\title{
Fluorescence of silicon nanoparticles suspended in water: reactive co-deposition for the control of surface properties of clusters
}

\author{
K. von Haeften, A. Akraiam, G. Torricelli and A. Brewer \\ Department of Physics and Astronomy, University of Leicester, Leicester, LE1 7RH, United \\ Kingdom
}

\begin{abstract}
Fluorescent silicon nanoparticles have been produced in a two-step process in ultra high vacuum. First, silicon clusters were produced in the gas phase in a molecular beam. At the end of the cluster beam machine the cluster were co-deposited with water onto a cold target. Melting of the ice yields a suspension that fluoresces at $420 \mathrm{~nm}$ when excited with ultraviolet light. The fluorescence intensity remains constant over a period of more than a year. Photo-absorption and photo-luminescence spectra provide evidence of a $\mathrm{Si} / \mathrm{SiO}_{2}$ core-shell structure having a silicon core size of at least $1.4 \mathrm{~nm}$ in diameter and oxygen deficient $\mathrm{O}-\mathrm{Si}-\mathrm{O}$ defects as the origin of the deep-blue fluorescence. Furthermore, the fluorescent suspension was deposited on freshly cleaved highly oriented pyrolytic graphite (HOPG). AFM images recorded in UHV showed networks of agglomerated clusters, their smallest units having a diameter of typically $0.7 \mathrm{~nm}$.
\end{abstract}

Keywords: Optical properties of clusters, Fluorescent silicon, Photoluminescence, properties and materials, Nanocrystals, nanoparticles, and nanoclusters, Methods of materials synthesis and materials processing, Reactions in clusters

PACS: $36.40 . \mathrm{Vz}, 78.55 .-\mathrm{m}, 78.67 . \mathrm{Bf}, 81.20 .-\mathrm{n}, 82.33 . \mathrm{Fg}$

\section{INTRODUCTION}

Fluorescent silicon nanoparticles have recently regained considerable attention because of their relevance to resolving a number of technological key challenges, such as (i) the fabrication of ultra high-dense integrated electronic circuits for which optical signal transmission has been suggested as a remedy to overcome fundamental speed-limitations imposed with electron transport [1], (ii) an increase in efficiency of solar cells by conversion of a larger spectral range into electrical energy [2, 3] and (iii) labelling and marking in bio-medicine. The latter is particularly interesting as silicon-based nanoparticles could provide an alternative to commonly used luminescent III-V semiconductor quantum dot labels, such as CdTe, as these are of limited use for in-vivo applications due to their toxicity. $\mathrm{Si}$ and $\mathrm{SiO}_{2}$ are so far regarded as harmless.

This paper is concerned with a novel production route for fluorescent Si nanoparticles. This route provides flexibility as two requirements to achieve luminescent Si - confinement and surface passivation - are achieved independently in separate steps. Furthermore, the method yields the nanoparticles within an aqueous suspension which is a great advantage for bio-medical applications. Within the aqueous environment the nanoparticles show remarkable stability in fluorescence intensity. Over a trial period of a year, practically no loss in fluorescence intensity has been observed. We show that the fluorescence is most likely attributed to small $\mathrm{Si} / \mathrm{SiO}$ units, of about $1 \mathrm{~nm}$ in diameter. 


\section{PRODUCTION OF NANOPARTICLES}

Our production method achieves confinement and passivation in a two-step process. Si is an indirect semiconductor and a requirement to increase its fluorescence yield is to provide confinement. Confinement is naturally achieved in clusters comprising a few tens up to thousands of $\mathrm{Si}$ atoms and the specific advantage of using clusters is that the degree of confinement can be controlled by changing the cluster-size. To produce $\mathrm{Si}$ clusters we used a gas-aggregation source in which $\mathrm{Si}$ is sputtered from a p-doped target. The principles of such a machine have been described in the literature $[4,5,6,7]$. We used Ar as sputter gas and mixtures of Ar and He to control the aggregation process and thus the cluster size. Neutral and charged clusters are produced [8].

Downstream of the aggregation zone the clusters passed through several differential pumping stages until they hit a liquid nitrogen-cooled target, together with a continuous flow of water vapour, leading to the build-up of an ice layer with Si clusters enclosed. A schematic of the process is shown in figure 1. After deposition, which typically lasted 30 minutes the UHV chamber was vented with 99.995-graded nitrogen and the target was warmed up so that the ice melted and the suspension dropped into a dish.

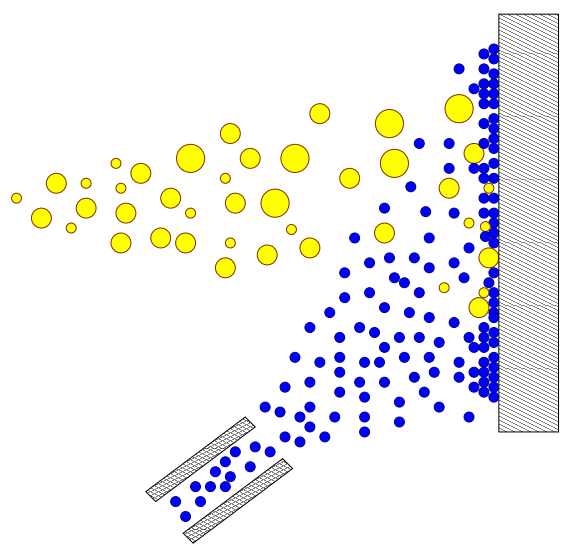

FIGURE 1. Schematic of the silicon clusters and water co-deposition process.

This second step is crucial to provide efficient passivation, another requirement for achieving fluorescent $\mathrm{Si}$, as free clusters exhibit dangling bonds at the surface at which excitations can decay fast and non-radiatively. Passivation with, for instance $\mathrm{SiO}_{2}$, closes these pathways and allows the excited clusters to assume their ground state through a radiative process. We have shown that the treatment with water is mandatory for achieving efficient luminescence [9]. Clusters, directly deposited onto quartz and left in air to oxidise showed no fluorescence. We believe that $\mathrm{SiO}_{2}$ is formed within a reaction between, presumably ionic, Si clusters and water, similarly to nano-oxidation when using charged AFM tips $[10,11,12]$. 


\section{SPECTROSCOPY, IMAGING AND NANOPARTICLE SIZE}

To characterise the origin of the fluorescence and the size of the nanoparticles we recorded UV/VIS absorption spectra as well as photoluminescence spectra at different excitation wavelengths. Figure 2 shows the optical absorption of the liquid sample (dotted line) as well as the fluorescence for 240 and $305 \mathrm{~nm}$ excitation. The absorption spectrum shows a band gap and an increase in absorption for wavelengths shorter than $350 \mathrm{~nm}$. The magnitude of the band gap energy is smaller than that of $\mathrm{SiO}_{2}$ indicating the presence of another absorber. The rapid increase at about $275 \mathrm{~nm}$ can be explained by light absorption of silicon nanoparticles with diameters less than $1.5 \mathrm{~nm}$ due to quantum confinement [13]. The prominent band at $420 \mathrm{~nm}$ was assigned to $\mathrm{T}_{1} \rightarrow \mathrm{S}_{0}$ defect fluorescence of twofold coordinated silicon $(\mathrm{O}-\mathrm{Si}-\mathrm{O})$ present in a $\mathrm{SiO}_{2}$ layer at the surface of the nanoparticles. This assignment is based on the similarity to the fluorescence of selected defect-rich $\mathrm{SiO}_{2}[14,15,16]$, however, the excitation proceeds via a previously unreported state at $308 \mathrm{~nm}$, presumably an interface exciton [9]. For excitation at $240 \mathrm{~nm}$ fluorescence emerges only from states lower that $275 \mathrm{~nm}$, presumably the HOMO state of quantum confined $\mathrm{Si}$, confirming our size estimation.

To further investigate the nanoparticle size we cleaved a HOPG crystal, placed a drop of the fluorescent suspension on it, evacuated subsequently and recorded AFM images.

We identified regions with different nanoparticle densities. A typical image from a less-dense region is shown in figure 3. The nanoparticles agglomerate and, in this density regime, form preferentially $1 \mathrm{D}$ networks. Single clusters can be identified, the smallest

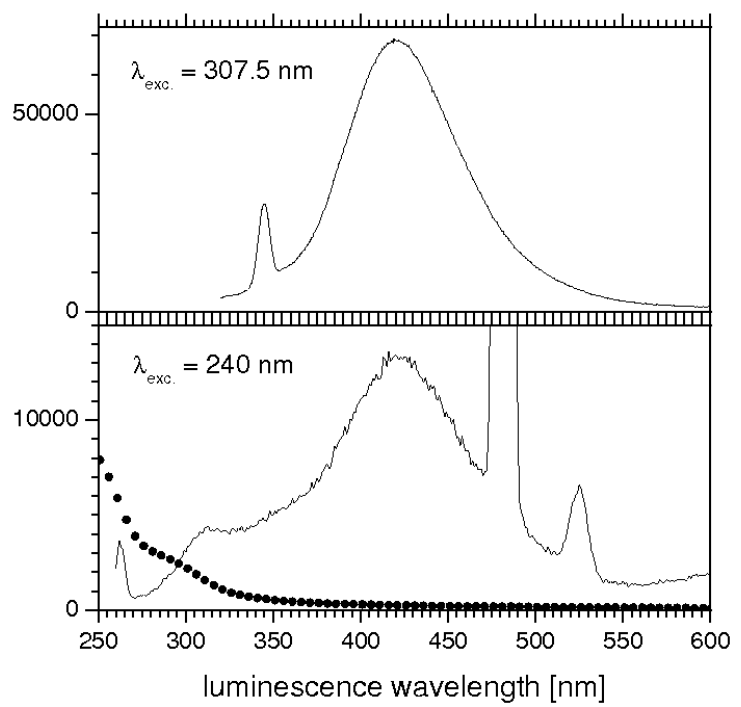

FIGURE 2. Fluorescence spectra of silicon nanoparticles suspended in water recorded at three different excitation wavelengths. For comparison the UV/VIS absorption spectrum is shown (dotted line). The intensity scale for this spectrum is in arbitrary units. 


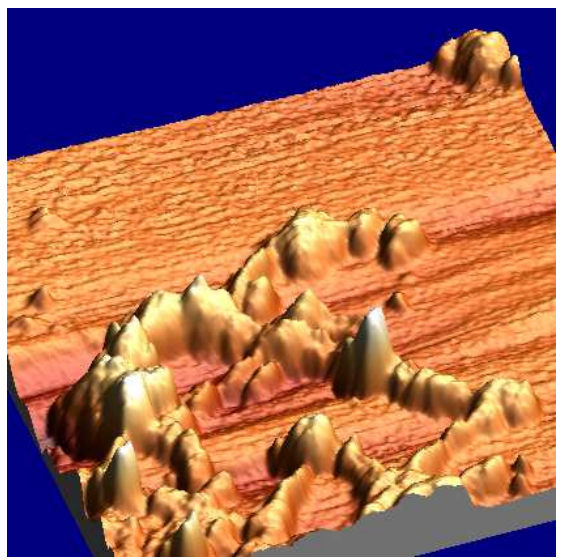

FIGURE 3. AFM image of nanoparticles after deposition of a drop of the suspension on freshly cleaved HOPG.

showing a lateral dimension of $8 \mathrm{~nm}$ in diameter and a height of $0.7 \mathrm{~nm}$. We ascribe the lateral extension to the convolution with the AFM tip. The height of the smaller clusters is in fair agreement with the size derived from our spectroscopic analysis and it is very possible that the fluorescence stems from these small clusters.

The authors are grateful to Chris Binns and Mark Lowe for the provision of experimental equipment and to the Royal Society for support through a Research Grant.

\section{REFERENCES}

1. L. Pavesi, J. Phys. C 15, 1169-+ (2003).

2. V. Švrček, A. Slaoui, and J. Muller, Thin Solid Films 451, 384-388 (2004).

3. M. Stupca, M. Alsalhi, T. Al Saud, A. Almuhanna, and M. Nayfeh, Appl. Phys. Lett. 91, 063107 (2007).

4. H. Haberland, M. Karrais, and M. Mall, Z. Phys. D 20, 413-415 (1991).

5. M. Astruc Hoffmann, G. Wrigge, B. v Issendorff, J. Müller, G. Ganteför, and H. Haberland, Eur. J. Phys. D: At., Mol. Clusters 16, 9-11 (2001).

6. S. Pratontep, S. Carroll, C. Xirouchaki, M. Streun, and R. Palmer, Rev. Sci. Instr. 76, 045103 (2005).

7. K. Wegner, P. Piseri, H. Tafreshi, and P. Milani, J. Phys. D: Appl. Phys. 39, R439-R459 (2006).

8. K. von Haeften, C. Binns, A. Brewer, O. Crisan, P. Howes, M. Lowe, C. Sibbley-Allen, and S. Thornton, Eur. Phys. J. D 52, 11-14 (2009).

9. A. Brewer, and K. von Haeften, Appl. Phys. Lett. 94, 261102 (2009).

10. M. Tello, and R. Garcia, Appl. Phys. Lett. 79, 424 (2001).

11. H. Kuramochi, K. Ando, and H. Yokoyama, Surf. Sci 542, 56-63 (2003).

12. D. Stiévenard, and B. Legrand, Progress in surface science 81, 112-140 (2006).

13. N. Hill, and K. Whaley, Phys. Rev. Lett. 75, 1130-1133 (1995).

14. L. Skuja, A. Streletsky, and A. Pakovich, Solid State Commun. 50, 1069-1072 (1984).

15. L. Skuja, J. Non-Cryst. Solids 149, 77-95 (1992).

16. H. Nishikawa, T. Shiroyama, R. Nakamura, Y. Ohki, K. Nagasawa, and Y. Hama, Phys. Rev. B 45, 586-591 (1992). 